\title{
Channel assignment using differential evolution algorithm in cognitive radio networks
}

\author{
Shahzad Latif $1,2, *$, Suhail Akraam ${ }^{1}$, Muhammad Aamer Saleem ${ }^{3}$ \\ ${ }^{1}$ School of Engineering and Applied Sciences, ISRA University, Islamabad, Pakistan \\ ${ }^{2}$ School of Engineering and Applied Sciences, SZABIST, Islamabad, Pakistan \\ ${ }^{3}$ Hamdard Institute of Engineering and Technology, Hamdard University, Islamabad, Pakistan
}

\section{A RT ICLE INFO}

Article history:

Received 7 May 2017

Received in revised form

20 July 2017

Accepted 25 July 2017

Keywords:

Cognitive radio networks

Differential evolution algorithm

Channel assignment

Optimization

\begin{abstract}
A B S T R A C T
The emerging wireless applications have increased the demand of wireless spectrum significantly. Present spectrum assignment is static, due to which problem of spectrum scarcity has been raised. Cognitive Radio (CR) is a promising technology to deal with spectrum scarcity problem, which uses dynamic spectrum allocation to utilize the vacant spectrum. The CR intelligently scans the spectrum in its vicinity and search the vacant spectrum. The optimization of available spectrum is important research challenge in cognitive radio networks (CRNs). In this research work, we have optimized the spectrum utility of SUs using Differential Evolution (DE) algorithm in order to reduce the interference incurs to primary users (PUs) and as well as among the secondary users (SUs). Moreover, the results are compared with other evolutionary channel assignment algorithms like Fuzzy Logic Ant Colony System (FLACS) and Color Sensitive Graph Coding Method (CSGC). It has been observed that the results of proposed algorithms can further enhance the spectrum utility in CRNs in comparison to FLACS and CSGC.
\end{abstract}

(C) 2017 The Authors. Published by IASE. This is an open access article under the CC BY-NC-ND license (http://creativecommons.org/licenses/by-nc-nd/4.0/).

\section{Introduction}

Currently the requirement for wireless spectrum has been increased drastically (FCC, 2013). It is predicted that spectrum demand will increases 10 times between 2015 to 2020 . In order to cope with this problem of spectrum scarcity, Cognitive Radio (CR) is a promising technology (Haykin, 2005). CRNs have functions: spectrum sensing; control; sharing and spectrum mobility (Akyildiz et al., 2006). This paper concentrates on how to optimize available spectrum among unlicensed users called SUs which is detected unoccupied by licensed users called primary users (PUs) in order to avoid interference between PUs and SUs, and also to avoid interference among SUs.

\section{Literature survey}

Excessive efforts of research exist in literature to solve the spectrum sharing problem in CRNs. There

\footnotetext{
* Corresponding Author.

Email Address: shahzadlatif97@gmail.com (S. Latif) https://doi.org/10.21833/ijaas.2017.08.023

2313-626X/C) 2017 The Authors. Published by IASE.

This is an open access article under the CC BY-NC-ND license

(http://creativecommons.org/licenses/by-nc-nd/4.0/)
}

are so many channel assignment algorithms and methods have been proposed on the basis of centralized or distributed architecture, overlay or underlay techniques of accessing spectrum and cooperative or non-cooperative allocation behavior of spectrum. These proposed methods include game theory (Nie and Comaniciu, 2006), auction and pricing mechanism (Kloeck et al., 2005; Huang et al., 2006), graph coloring (Peng et al., 2006) and local bargaining (Cao and Zheng, 2005). The stochastic searching methods which have been inspired by social behavior of species and natural evolutions are referred as evolutionary algorithms (Saxena and Kothari, 2016). These algorithms have proved that they work more efficiently while dealing with discontinuous, noisy and differential problems. Evolutionary Computation (EC) applies parallel search in search space. EC does not use any derivative information for space searching; only fitness values from individuals are used for getting help in search space (Engelbrecht, 2006).

In Zhao et al. (2009) Genetic Algorithm (GA) and Particle Swarm Optimization (PSO) based spectrum assignment algorithms were investigated in order to assign spectrum in CRNs. Zhao et al. (2009) claimed that PSO algorithm can be used to find an optimal solution under the three specific objective functions 
introduced in Peng et al. (2006). However, the performance of these algorithms versus different PUs, SUs and channels available are not well investigated. In Koroupi et al. (2013), a fuzzy based ant colony approach is used to analyze the spectrum assignment versus PUs, SUs and available channels.

The solution represented in Koroupi et al. (2013) improves the performance further. Contribution of this paper is that the DE based spectrum assignment algorithm is proposed which further enhances the performance spectrum utility in comparison with fuzzy logic inspired ants algorithm by Koroupi et al. (2013) and Color Sensitive Graph Coloring (CSGC) method by Peng et al. (2006).

GA has diversity in population but it is slow, on the other hand PSO is speedy but it has less diversity. DE has both the features; it is speedy as well as diversity. DE algorithm has a balance between diversity and exploration (Devi et al., 2014; Zhou et al., 2011). This paper is organized as follows: in section 3 Network model is discussed. Section 4 proposed DE based Channel Assignment Algorithm is discussed. Section 5 presents simulation results and discussion. Finally the conclusion is drawn in section 6.

\section{Network model for spectrum assignment}

Open system model (Peng et al., 2006) is considered in this paper. SUs use bands which are licensed, provided that there is no interference as imposed by PUs. SUs are able to detect PUs by accessing a central database. Recently, a report of FCC (FCC, 2013) points towards the feasibility to allow SUs operations in TV broadcast white spaces in time and location. We describe the model by the following example.

Fig. 1 illustrates the example of deployment in which an inactive broadcast television, which is a PU $x$, utilizes the spectrum in order to provide residential community connections via a wireless connection.

In this example, SUs are marked as 1, 2, 3 and 4 . PU $x$ acquires only $m^{\text {th }}$ channel with its associated protection area of region $d_{p}(x, p)$. Any kind of radiation from the side of SUs falling in this region will interfere the PU.

Let $n^{\text {th }}$ SU can adjust its range of interference into $d_{S}(n, m)$ by tuning transmission power on $m^{\text {th }}$ channel in order to avoid interfering with the PU. Here, we are assuming that $n^{\text {th }}$ SU can use the $m^{\text {th }}$ channel as neighbor of PU $x$, provided that the distance fulfills the following expression (Eq. 1):

$d_{S}(n, m) \leq \operatorname{Dist}(n, x)-d_{p}(x, m)$.

In the above expression, the distance between PU $x$ and SU $n$ is represented as $\operatorname{Dist}(n, x)$. Generally, range of interference $d_{S}$ is bounded between maximum and minimum transmission power which is $\left[d_{\text {min }}, d_{\text {max }}\right]$, so $d_{S}$ is considered as the transmission range of SU. It is assumed that channels are orthogonal to each other.

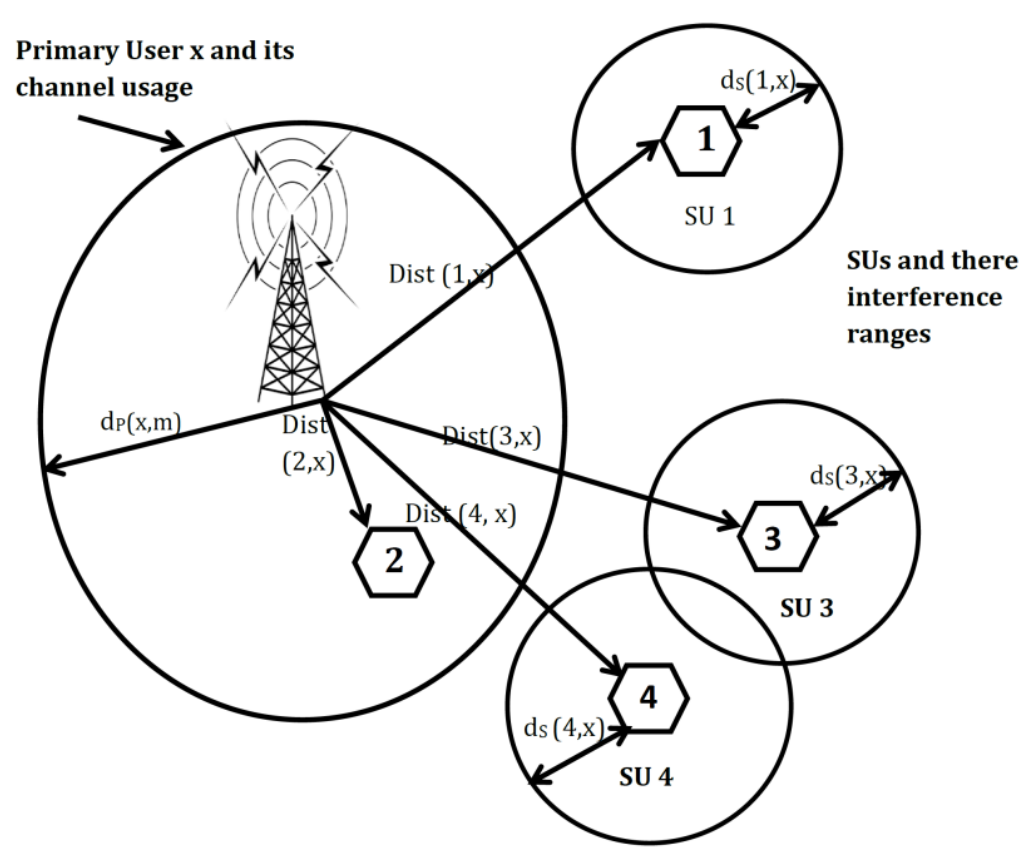

Fig. 1: Cognitive radio network model

Effective fairness and utility are key objectives in open spectrum system. Above two goals combine to form utility functions which can be customized for every kind of wireless network applications. Three types of utility functions described in Peng et al. (2006) are studied and compared for these objectives. It is assumed that location of users and spectrum is static during channel assignment process.

It is assumed that network model has a fixed topology for a sufficient time. Wireless network consists of $X$ PUs and $N$ SUs which compete for $M$ channels available. The main components of this network model are defined as follow. 


\subsection{Matrix of channel availability}

$L=\left[\left\{l_{n, m}\right\} \in(0,1)\right]_{N \times M}$. If $l_{n, m}=1, m^{\text {th }}$ channel is available for $n^{\text {th }}$ SU. Otherwise $m^{\text {th }}$ channel is not available for $n^{\text {th }}$ SU and it is under use of PU.

\subsection{Matrix of channel rewards}

$B=\left\{b_{n, m}\right\}_{N \times M}$. This represents rewards of $n^{\text {th }} \mathrm{SU}$ which occupies $m^{\text {th }}$ channel. Usually, users are using maximum throughput or bandwidth assuming no interference from neighbor users. The $n^{\text {th }} \mathrm{SU}$ can get the reward $b_{n, m}=d_{S}^{2}(n, m)$ which fulfill the criteria $d_{\min } \leq d_{S}(n, m) \leq d_{\max }$ (Peng et al., 2006).

\subsection{Interference constraint matrix}

$C=\left[\left\{c_{n, k, m}\right\} \in(0,1)\right]_{N \times N \times M}$ three dimensional matrix represents the interference constraint among SUs. If $c_{n, k, m}=1$ then $n^{\text {th }}$ and $k^{\text {th }}$ SUs would interfere with each other for obtaining the same $m^{\text {th }}$ available channel. If $c_{n, k, m}=0$ then $n^{\text {th }}$ and $k^{\text {th }}$ SUs not interfering with each other for the same $m^{\text {th }}$ channel.

\subsection{Matrix of channel assignment without conflict}

$A=\left[\left\{a_{n, m}\right\} \in(0,1)\right]_{N \times M}$. If $a_{n, m}=1$, it means that $m^{\text {th }}$ channel has been already assigned to $n^{\text {th }} \mathrm{SU}$ and vice versa. It must meet following constraint of interference which is defined by matrix $C$ as follows (Eq. 2):

$a_{n, m} \times a_{k, m}=0$ if $c_{n, k, m}=1$

Eq. 2 represents that if two SUs qualify for the same channel then discards one of them. Let $\Lambda(\mathrm{L}, \mathrm{C})_{N, M}$ represent the conflict free channel assignment set for $N$ SUs, $M$ channels and interference constraints $C$. For a given conflict free channel assignment, $n^{\text {th }}$ user reward is defined as $r_{n}=\sum_{m=1}^{M} a_{n, m} \times b_{n, m}$.

We use $R=\left\{r_{n}=\sum_{m=1}^{M} a_{n, m} \times b_{n, m}\right\}_{N \times 1}$ as a reward vector for a given channel assignment that each user gets. Objective here is to increase spectrum utility which is represented as $U(R)$.

By considering the above mentioned model, the problem of allocating spectrum is defined by optimization problem as follows (Eq. 3):

$A^{*}=\max _{A \in L, C} U(R)$

In the above expression, $A^{*}$ is conflict free optimal matrix of assigning channel. We have considered following three objective functions (Peng et al. 2006):

\subsubsection{Maximum sum reward (MSR)}

Our objective here is to maximize overall secondary users rewards irrespective of fairness for each SU considering the interference constrain (Eq. 4).

$U(R)=\sum_{n=1}^{N} r_{n}$

\subsubsection{Max min reward (MMR)}

Our objective here is to maximize the reward of those SUs which got the least reward in wireless spectrum. In CRN, some selfish SUs can get the maximum spectrum bands so in order to increase the reward of those SUs which obtain least reward (Eq. 5).

$U(R)=\min \left(r_{n}\right) \quad \forall(1 \leq n \leq N)$

\subsubsection{Max proportional fair (MPF)}

Our objective here is equality among SUs irrespective of reward that they got in order to balance the spectrum opportunities among SUs (Eq. 6).

$U(R)=\sum_{n=1}^{N} \log \left(r_{n}\right)$

The above fairness function can also be written as (Peng et al., 2006):

\subsection{Proposed differential evolution based spectrum assignment algorithm}

Number of solutions which are possible inside search space are very large, therefore exhaustive search techniques (Michalewicz and Fogel, 2013) cannot be applied for all states in practical applications. Since CRN works in a real time system, so to find an optimal channel assignment is essential. We have proposed the use of Differential Evolution algorithm to get optimal solution (Eq. 7).

$U(R)=\left(\prod_{n=1}^{N}\left(r_{n}+10^{-6}\right)\right)^{1 / N}$

DE is a population based search algorithm. The main difference in reproduction step, new population is created using an arithmetic operation among randomly selected three individuals (parents) of current population. For each parent $y_{i}(t)$ create an offspring $y_{i}^{\prime}(t)$ for $t$ generations in the following way. Randomly select three parents $y_{i_{1}}(t), y_{i_{2}}(t)$ and $y_{i_{3}}(t)$ such that $i_{1} \neq i_{2} \neq i_{3} \neq i$ and $i_{1}, i_{2}, i_{3}$ belongs to population size. Select a random number $q \in\left(1,2,3 \ldots n_{x}\right)$ where $n_{x}$ number of parameters (genes) of a single chromosome. The probability of reproduction represented by $P_{r} \in$ $[0,1]$. Then define a threshold value randomly between $(0,1)$. If $P_{r}$ is less than this threshold value, or if $j=q$, let (Eq. 8)

$y_{i j}^{\prime}(t)=y_{i_{3} j}(t)+\beta\left\{y_{i_{1 j}}(t)-y_{i_{2} j}(t)\right\}$,

otherwise, let (Eq. 9)

$y_{i j}^{\prime}(t)=y_{i j}(t)$. 
In the above expression, $\beta \in(0, \infty)$ is an amplifying factor, $y_{i j}^{\prime}(t)$ and $y_{i j}(t)$ represents respectively offspring and parent of $j^{\text {th }}$ parameter. In DE even when $P_{r}=0$ at least one parameter differs from in offspring because $j=q$. In DE above reproduction process require that population size $>$ 3.

\subsubsection{Encoding mechanism}

Now discuss the encoding of DE algorithm for spectrum allocation problem. Each chromosome represents a solution for SU and its fitness value related to utility functions defined above. We consider utility functions as objective functions. DE consists of chromosomes. A chromosome further divided into parameters also called genes represented in binary form. For the problem of $M$ channels and $N$ SUs, SUs are represented by $N$ chromosomes, and channels are represented by $M$ genes in a chromosome as shown in Fig. 2. The allocated channels for a SU are represented by binary bits. If bit value is one then corresponding channels is allocated to SU and vice versa.
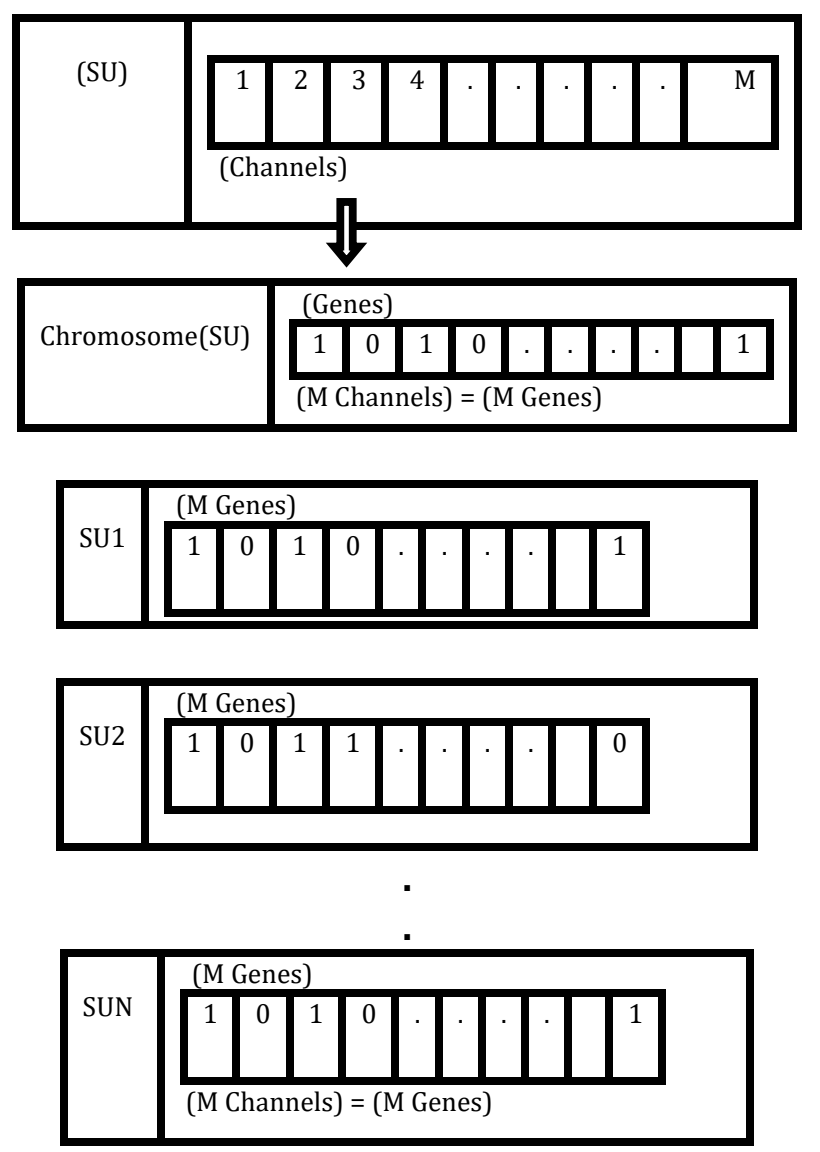

Fig. 2: DE chromosome mapping for channels allocation

Population consists of SUs. Initialized the population and conflict free channels assignment matrix $A$ is constructed according to criteria defined in Eqs. 1 and 2. If the two SUs occupy the same channel as shown in Fig. 3, then set one of SUs channel bit value to zero to avoid conflict.

Then offspring is created using Eqs 8 and 9. After this step, fitness of initial population and offspring population is calculated using objective functions defined in Eqs 5, 6, and 7. If the fitness of offspring better than the parent then it is replaced with it, otherwise parent is carried over to the new population. The iteration process continued up to pre-defined number of cycles.

\begin{tabular}{|c|c|c|c|c|c|c|c|c|c|c|}
\hline Channels & 1 & 2 & 3 & 4 & 5 & 6 & 7 & 8 & 9 & 10 \\
\hline \multirow{2}{*}{ SU 1 } & 1 & 0 & 1 & 0 & 0 & 0 & 1 & 1 & 1 & 0 \\
& 4 & & & & & & 位 & $\mathbf{4}$ & $\mathbf{4}$ & \\
\hline SU 2 & 1 & 0 & 0 & 0 & 1 & 1 & 1 & 1 & 1 & 0 \\
\hline
\end{tabular}

Fig. 3: Infeasible allocation of SU1 and SU2

\section{Results and discussions}

We are considering a wireless network of $10 \times 10$ region, simulated $X$ number of PUs by placing them randomly in this area. Pus of this class can serve as base stations for different kind of wireless networks operator. Then, we deploy $N$ SUs randomly in this network. By following the method of Peng et al. (2006), Koroupi et al. (2013), SUs are assumed to be identical. We take different ranges for the PUs, SUs and available channels. The parameters values represented in Table 1 are considered to compare results with results of papers (Peng et al., 2006; Koroupi et al., 2013). Spectrum of PUs and SUs are allocated randomly between values 0 to 10 . Maximum ten numbers of channels $\left(C_{\max }\right)$ can be assigned to a SU.

Table 1: Parameter values

\begin{tabular}{cc}
\hline Parameter & Values \\
\hline $\mathrm{C}_{\max }$ & 10 \\
$\mathrm{~d}_{\mathrm{P}}$ & 2 \\
$\mathrm{~d}_{\min }$ & 1 \\
$\mathrm{~d}_{\min }$ & 4 \\
$\mathrm{P}_{\mathrm{r}}$ & 0.5 \\
$\beta$ & 0.5 \\
\hline
\end{tabular}

\subsection{Impact by changing the number of channels}

Fig. 4a quantifies the performance of proposed algorithms for MSR as the number of channels changes for $N=10$, and $X=20$ We observed that in general all three utilities increases as the number of channels increases. In comparison with FLACS, DE and GA perform better up to $M=5$ to 17 and $M=$ 25 to 30 channels DE slightly better for $M=30$ channels. Fig. 4b, for MMR, DE outperform than FLACS for total range of channels, GA perform better that FLACS-SA in the range $M=25$ to 30. Fig. 4c, for MPF, DE outperform for all range $M=5$ to $M=30$ channels.

\subsection{Impact by varying number of SUs}

Next analyze the performance of all proposed algorithms with Koroupi et al. (2013), increasing number of secondary users clearly creates additional interference constraints. Set $M=10, X=20$. Figs. $5 a, 5 b$, and $5 c$ show each one three utilities degrade as the number of SUs increases. Fig. 5a with MSR 
utility, DE-SA perform better than FLACS-SA, BCO performance good for initial SUs $N=5$ to 7. Fig. $5 \mathrm{~b}$ for MMR, DE perform better than FLACS, GA also show better performance than FLACS for $M=$ 5 to 10. Fig. $5 \mathrm{c}$ for MPF, DE performs good for whole range of increasing SUs.

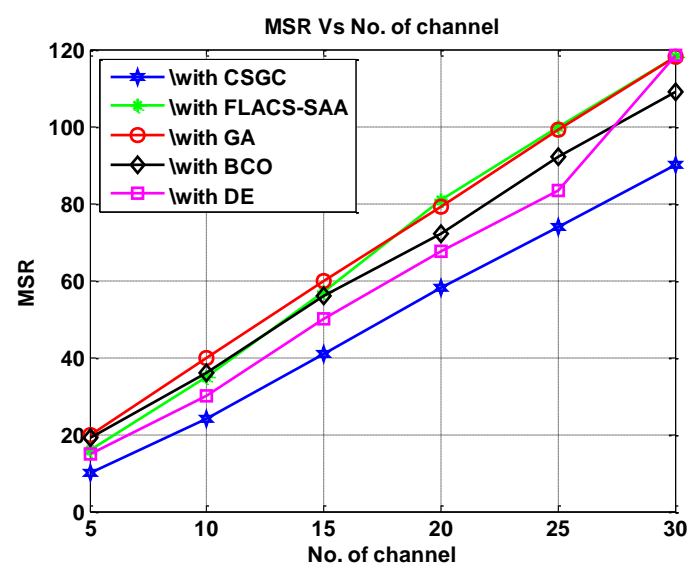

(a)

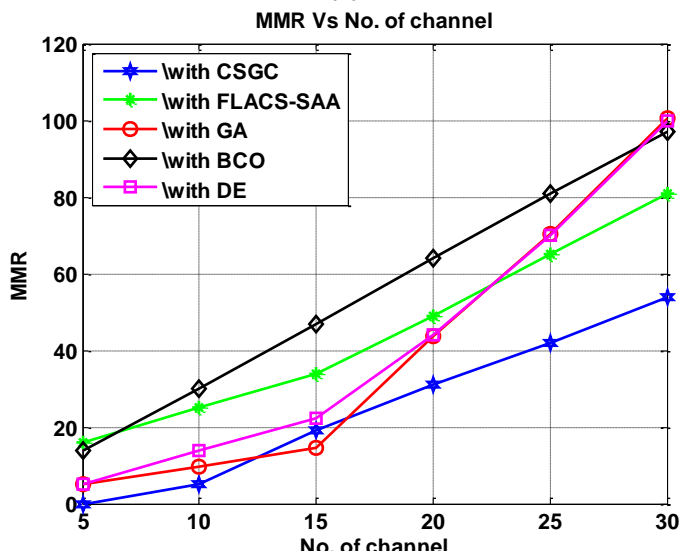

(b)

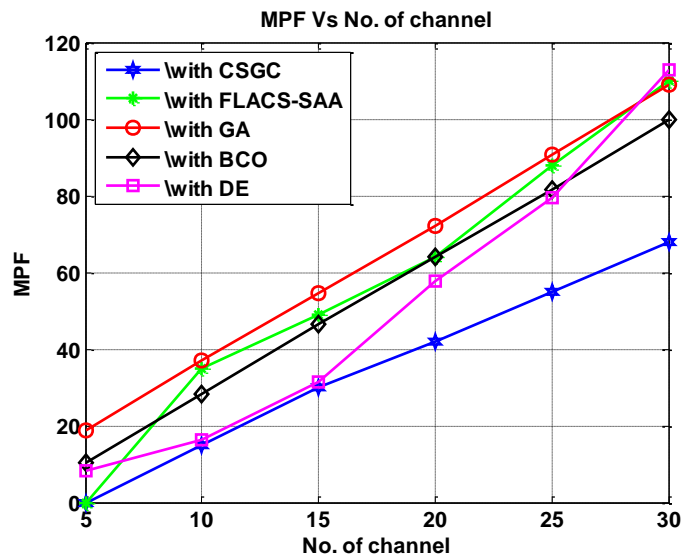

(c)

Fig. 4: Number of channels versus (a) MSR, (b) MMR, (c) MPF

\subsection{Impact by varying number of PUs}

Similarly, increasing the number of primary users could lessen all three utilities. Fig. 6a for MSR, proposed algorithm not performs well in this case. Fig. $6 \mathrm{~b}$ represent MMR versus Number of primary users, DE perform better than FLACS for $X=5$ to 25, for $X=25$ to 50 DE and FLACS both perform approximately equally well.

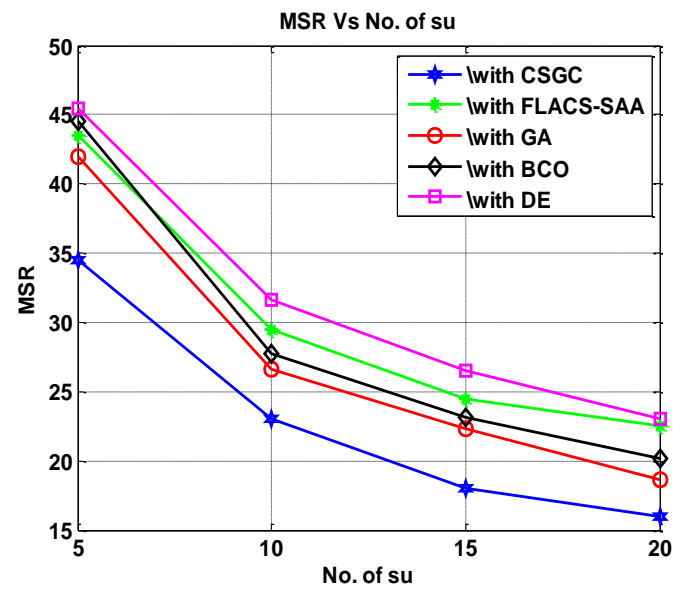

(a)

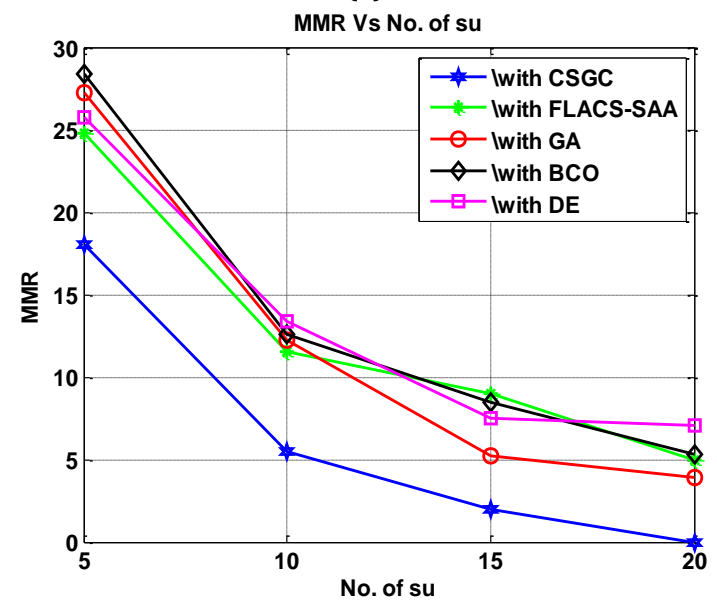

(b)

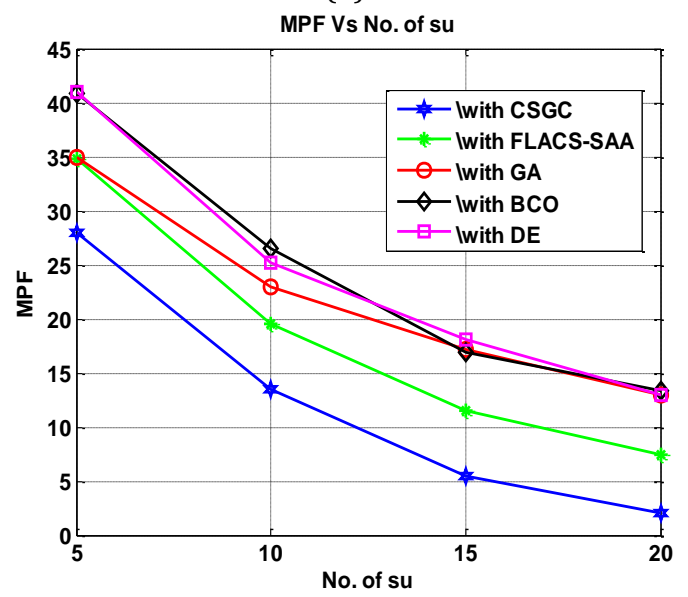

(c)

Fig. 5: Number of SUs versus (a) MSR, (b) MMR,(c) (MPF)

BCO, GA perform well for $X=1$ to 10 and perform equal to FLACS for $X=50$. Fig. $6 \mathrm{c}$ represent MPF utility versus $X$, DE perform better than FLACS for $X=30$ to 50 , DE and FLACS perform equally well for $X=30$ to 50 . BCO, GA performance equal to FLACS for $X=5$ primary users only.

\subsection{Comparison among three utilities}

Fig. 7a represents comparison among all three utilities versus number of increasing channels. Fig. $7 \mathrm{~b}$ represents comparison among all three utilities versus number of increasing SUs. Fig. 7c represents 
comparison among all three utilities versus number of increasing PUs. MSR gives maximum utility in all three utilities, MPF give second best utility and MMR gives the least utility as it maximize the utility of min utilized user.

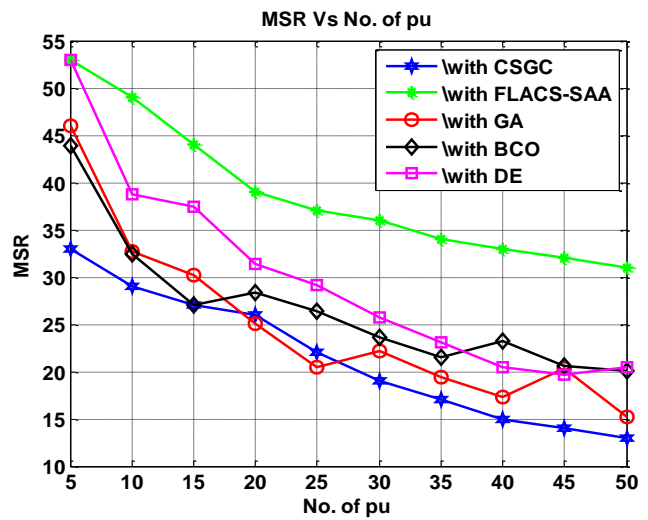

(a)

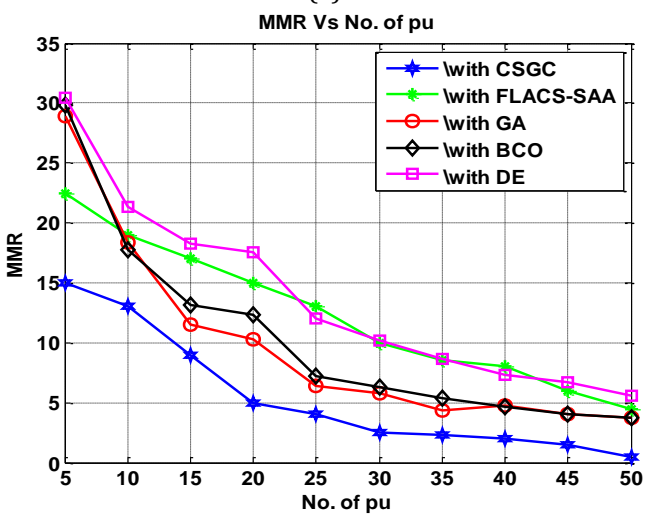

(b)

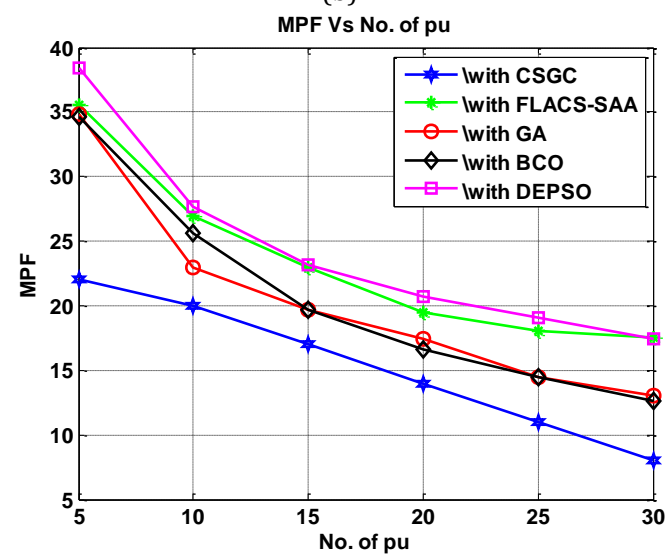

(c) (MPF)

Fig. 6: Number of PUs versus (a) MSR, (b) MMR, (c)

Table 2 represents average reward of MSR, MMR and MPF with increasing number of iterations for $N=20, M=20$ to compare it with results of Koroupi et al. (2013). DE performs better than FLACS for all three utilities.

\section{Conclusion}

The cognitive radio communication is a promising technology to deal with spectrum scarcity problem. In CRNs, the main objective is to increase the spectrum utility of SUs considering the interference avoidance incurs to primary users. In this research work, we have observed performance of proposed algorithm and its impact on a variety of factors such as increasing number of PUs, SUs, available channels, iterations and primary user protection area. The simulation results show that the proposed algorithm can enhance the performance of spectrum assignment of CRNs in comparison with the other studied algorithms. So, differential evolution based spectrum assignment model can be used to optimize wireless spectrum and also to avoid interference with primary users as well as among secondary users in cognitive radio networks. In future, we enhance this spectrum sharing problem to heterogeneous networks.

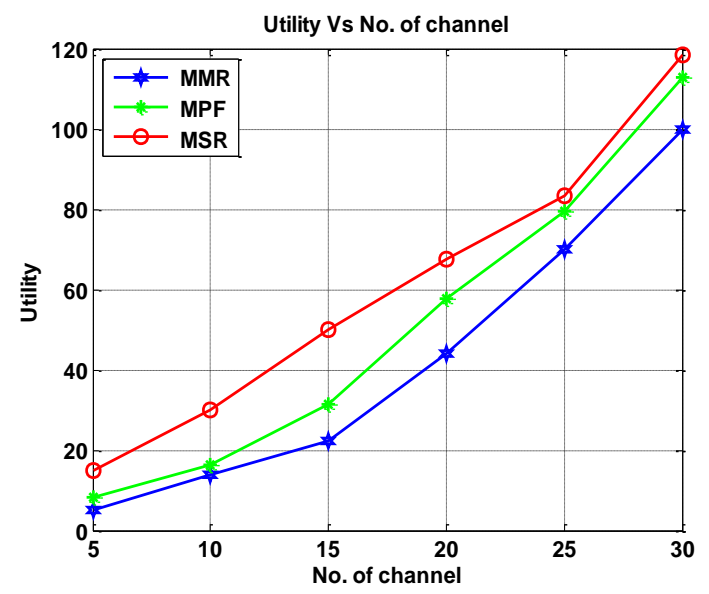

(a)

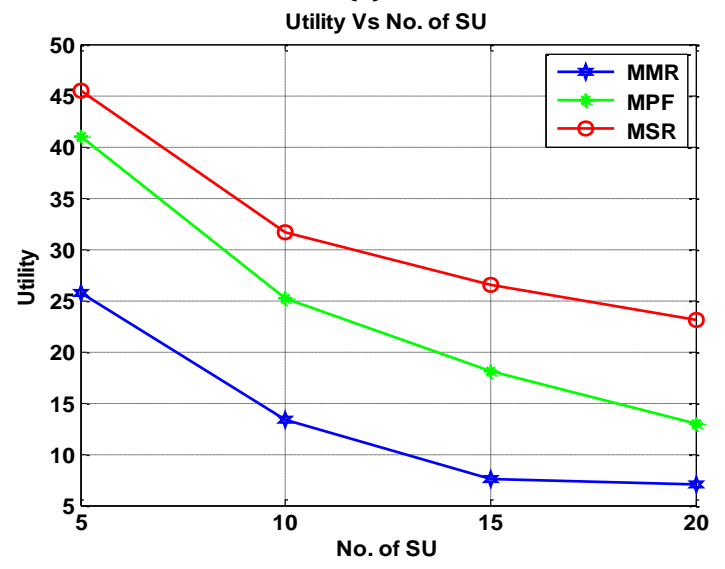

(b)

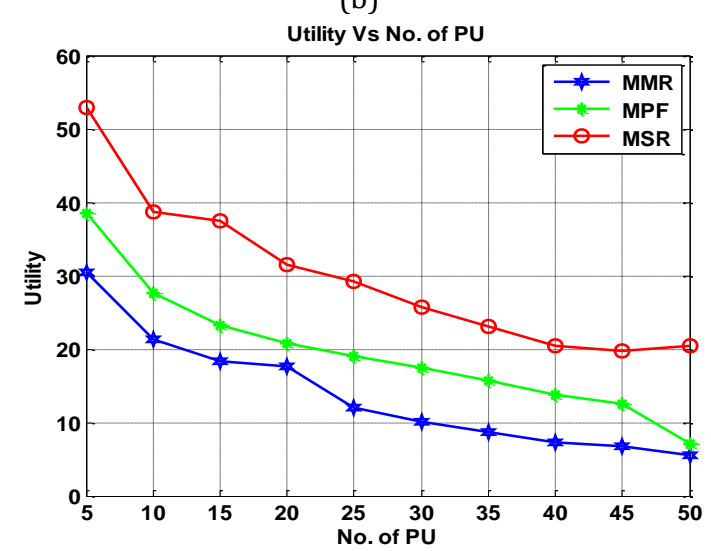

(c)

Fig. 7: Utility functions versus increasing (a) number of channels (b) SUs, (c) Pus 
Table 2: Number of Iterations of evolutionary algorithms in comparison with utility functions

\begin{tabular}{|c|c|c|c|c|}
\hline \multirow{2}{*}{$\begin{array}{c}\text { Iterations } \\
\text { (Generation) }\end{array}$} & \multirow{2}{*}{ Algorithm } & \multicolumn{3}{|c|}{ Average Reward(N=20, M= 20) } \\
\hline & & MSR & MMR & MPF \\
\hline \multirow{7}{*}{10} & GA-SAA & 1170.9525 & 2.3642 & 62.4950 \\
\hline & QGA-SAA & 1206.1508 & 9.5613 & 37.0068 \\
\hline & PSO-SAA & 1204.7035 & 8.3244 & 13.7659 \\
\hline & ACS-SAA & 1206.8396 & 9.3964 & 43.6243 \\
\hline & $\begin{array}{l}\text { FLACS- } \\
\text { SAA }\end{array}$ & 1211.4250 & 11.6402 & 58.7432 \\
\hline & CSGC & 1206.0437 & 2.7769 & 60.1252 \\
\hline & $\mathrm{DE}$ & 1244.1443 & 73.0000 & 258.2710 \\
\hline \multirow{7}{*}{50} & GA-SAA & 1229.1568 & 7. 2553 & 96.2020 \\
\hline & QGA-SAA & 1237.3000 & 39.5744 & 87.5857 \\
\hline & PSO-SAA & 1238.1758 & 28.0520 & 82.4346 \\
\hline & ACS-SAA & 1241.7378 & 42.7491 & 98.8839 \\
\hline & $\begin{array}{l}\text { FLACS- } \\
\text { SAA }\end{array}$ & 1252.4725 & 50.8461 & 103.6923 \\
\hline & CSGC & 1206.0437 & 2.7769 & 60.1252 \\
\hline & $\mathrm{DE}$ & 1332.7757 & 72.5000 & 258.2209 \\
\hline \multirow{7}{*}{300} & GA-SAA & 1238.9552 & 12.3750 & 116.7429 \\
\hline & QGA-SAA & 1238.9561 & $\begin{array}{c}56 . \\
2500\end{array}$ & 118.0215 \\
\hline & PSO-SAA & 1240.1890 & 50.9594 & 120.5298 \\
\hline & ACS-SAA & 1241.7826 & 65.9205 & 137.3048 \\
\hline & $\begin{array}{l}\text { FLACS- } \\
\text { SAA }\end{array}$ & 1248.9041 & 71.0437 & 145.9418 \\
\hline & CSGC & 1206.0437 & 2.7769 & 60.1252 \\
\hline & $\mathrm{DE}$ & 1512.3784 & 71.5000 & 258.2163 \\
\hline
\end{tabular}

\section{References}

Akyildiz IF, Lee WY, Vuran MC, and Mohanty S (2006). NeXt generation/dynamic spectrum access/cognitive radio wireless networks: A survey. Computer Networks, 50(13): 2127-2159.

Cao L and Zheng H (2005). Distributed spectrum allocation via local bargaining. In the Second Annual IEEE Communications Society Conference on Sensor and Ad Hoc Communications and Networks (SECON '05), IEEE, Santa Clara, USA: 475-486. https://doi.org/10.1109/SAHCN.2005.1557100

Devi R, Barlaskar E, Devi O, Medhi S, and Shimray R (2014). Survey on evolutionary computation tech techniques and its application in different fields. International Journal on Information Theory (IJIT), 3(3): 73-82.
Engelbrecht AP (2006). Fundamentals of computational swarm intelligence. John Wiley and Sons, Hoboken, USA.

FCC (2013). Before the federal communications commission (DA 16-274). Federal Communications Commission Washington, USA. Available online at: https://apps.fcc.gov/edocs_public/ attachmatch/DA-16-274A1.pdf

Haykin S (2005). Cognitive radio: Brain-empowered wireless communications. IEEE Journal on Selected Areas in Communications, 23(2): 201-220.

Huang J, Berry RA, and Honig ML (2006). Auction-based spectrum sharing. Mobile Networks and Applications, 11(3): 405-418.

Kloeck C, Jaekel H, and Jondral FK (2005). Dynamic and local combined pricing, allocation and billing system with cognitive radios. In the First IEEE International Conference on New Frontiers in Dynamic Spectrum Access Networks, IEEE, Baltimore, USA: 73-81. https://doi.org/10.1109/DYSPAN. 2005.1542619

Koroupi F, Salehinejad H, and Talebi S (2013). Spectrum assignment in cognitive radio networks using fuzzy logic empowered ants. Iranian Journal of Fuzzy Systems, 10(6): 119.

Michalewicz Z and Fogel DB (2013). How to solve it: Modern heuristics. Springer Science and Business Media, Berlin, Germany.

Nie N and Comaniciu C (2006). Adaptive channel allocation spectrum etiquette for cognitive radio networks. Mobile Networks and Applications, 11(6): 779-797.

Peng C, Zheng H, and Zhao BY (2006). Utilization and fairness in spectrum assignment for opportunistic spectrum access. Mobile Networks and Applications, 11(4): 555-576.

Saxena P and Kothari A (2016). Ant Lion Optimization algorithm to control side lobe level and null depths in linear antenna arrays. AEU-International Journal of Electronics and Communications, 70(9): 1339-1349.

Zhao Z, Peng Z, Zheng S, and Shang J (2009). Cognitive radio spectrum allocation using evolutionary algorithms. IEEE Transactions on Wireless Communications, 8(9): 4421-4425.

Zhou A, Qu BY, Li H, Zhao SZ, Suganthan PN, and Zhang Q (2011). Multiobjective evolutionary algorithms: A survey of the state of the art. Swarm and Evolutionary Computation, 1(1): 32-49. 